NOTICE WARNING CONCERNING COPYRIGHT RESTRICTIONS:

The copyright law of the United States (title 17, U.S. Code) governs the making of photocopies or other reproductions of copyrighted material. Any copying of this document without permission of its author may be prohibited by law. 


\title{
ABSTRACTION and VERIFICATION in ALPHARD: Design and Verification of a Tree Handler
}

\author{
Mary Shaw \\ Computer Science Department \\ Carnegie-Mellon University \\ Pittsburgh, Pa. 15213
}

June, 1976

\begin{abstract}
The design of the Alphard programming language has been strongly influenced by ideas from the areas of programming methodology and formal program verification. The interaction of these ideas and their influence on Alphard are described by developing a nontrivial example, a program for manipulating the parse tree of an arithmetic expression.

Keywords and Phrases: abstraction and representation, abstract data types, assertions, correctness, information hiding, program specifications, program verification, programming languages, programming methodology, structured programming,
\end{abstract}

The research described here was supported in part by the National Science Foundation (Grant DCR74-04187) and in part by the Defense Advanced Research Projects Agency (Contract F44620-73-C-0074, monitored by the Air Force Office of Scientific Research). 


\section{Contents}

Introduction ...........................................................................................................

Example: Minimal-Register Evaluation Order ......................................................... 4

Definition and Verification of a Form …............................................................ 7

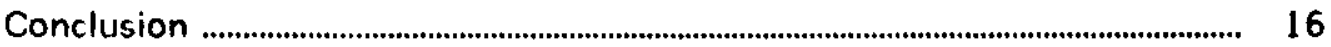

Appendix A: Complete Definition of Btree and Bnode ........................................ 18

Appendix B: Formal Definition of Graphs with Weighted Arcs ........................ 21

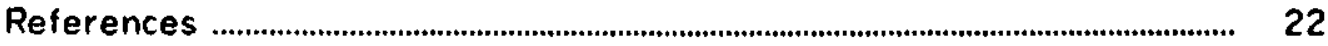




\section{Introduction}

The major concerns of the Alphard research are the total cost of software development and the quality of the resulting programs. Problems that arise from repeated modifications to
large programs, although often ignored in the literature, are of particular interest.

The Alphard language design has drawn heavily on previous work in both programming methodology and program verification. From the former we learned that in order to understand the programs we write, we must find some way to make them less complex; this may be done by restricting both the form of the programs (through modularity and localization of information [Parnas72]) and the process through which we create them (through stepwise precise, correct description of what a program does in order to use it without having to understand its implementation in detail; we also found techniques for writing and proving such
descriptions.

Our concern with modifiability implies that the things we do to reduce program complexity must remain visibly part of the program. Thus it is not sufficient to develop a program in a well-structured fashion; the structure that was imposed must be obvious in the Alphard the concept is realized through act data type has therefore become central. In derived from the Simula class [Dahi72] in language mechanism called a form. The form is and has the property that a programmer may revea same way as the CLU cluster [Liskov74], users while concealing details of the implementation.

This explicit distinction between the abstract behavior of a data type and the concrete adaptation, we show (a) that the concretestations correct [Hoare72]. In the Alphard abstract type, (b) that it is initialized properly, representation is adequate to represent the type both preserves the integrity of the representation that each operator provided for the terms of the abstract behavior and of the concretetion and does what it is claimed to do (in operator). The specific formulas that must be proved procedure that happens to implement the discussed in [Wulf76].

This paper describes the language and verification methodology that have resulted from merging these ideas. A particular example is used to motivate the description, and a independence of the abstract and concretel data abstraction was chosen to emphasize the

1 In this paper we will use the word "fype" in a nontechnical sense. In general, the abstraction introduced by a form need not be a type as we traditionally understand the word. 
Introduction

\section{Page 4}

for which a binary tree is a natural primitive data structure; the specifications and procedures for the solution assume the existence of an implementation of binary trees.

The Alphard form which defines those binary trees is developed in the third section. The development of that form is essentially independent of the motivating example, so the resulting abstraction is useful for other applications as well.

\section{Example: Minimal-Register Evaluation Order}

Suppose you are given an arithmetic expression represented as a binary parse tree and you are asked to output the nodes in postfix form with the subexpressions arranged in the order that minimizes the number of registers required for the expression evaluation. An algorithm for finding this order was given by Nakata; its description was refined by Johnsson [Nakata67, Johnsson75]. The algorithm has two steps:

Assign a weight $W$ to each node $n$ of the tree such that if $n$ is a leat then $W_{n}=0$, otherwise the immediate descendants of $n$ have labels right and left and $W_{n}=\min (\max (l e f t+1$, right $), \max (l e f t$, right +1$)) . W_{n}$ is the number of registers needed to evaluate the tree with root $n$.

To evaluate the expression, begin at the root node and walk through the tree generating code so that at each node the operand requiring the larger number of registers is evaluated first. If the operands require the same number of registers, the left operand is evaluated first. If the right operand is evaluated first, include an indication of the reversal in the output stream.

Assuming that suitable definitions for trees and an output stream exist, this is easily converted to a program. We will use a data abstraction called a btree as if it were a primitive data type. It acts like a binary tree with an associated collection of node references called bnodes. There are at least enough operators on bnodes to obtain the left son, the right son, and the value field (nodeval) of any node and to determine whether a node is a leaf. The btree form given in Appendix A provides other operators, but they are not required for this example. For convenience, we restrict the size of btrees. We will use a queve to construct the output; a suitable definition is given in [Wulf76].

We first write, more precisely than the English algorithm above, an expression that describes the desired output for a parse tree $E$. This expression appears as the post conditon (output assertion) of the procedure minreg that computes it. We let $W_{\text {left }}$ denote the weight of the left subtree, $W_{\text {rioht }}$ denote the weight of the right subtree, and invertop supply the operator that indicates subexpression reversal. ${ }^{2}$ The operator " $\sim$ " denotes concatenation. The 
Alphard: Design and Verification of a Tree Handler

Page 5

two-step algorithm is then written:

minreg(E: btree(?r: record(wt,data: integer), ?maxht: integer)) returns P: queue

post (isleaf $(E) \supset$ minreg $(E)=$ E.nodeval.data)

$\wedge\left(W_{\text {left }} \geq W_{\text {right }} \supset\right.$ minreg(E) $=$ minreg(E.leftson) $\sim$ minreg(E.rightson) E.nodeval.data)

$\wedge\left(W_{\text {left }}<W_{\text {right }} \supset\right.$ minreg $(E)=\operatorname{minreg}($ E.rightson $) \sim$ minreg(E.leftson)

$\sim$ invertop E.nodeval.data) $=$

begin local exptr: bnode(E);

markweights (exptr);

minregwalk(exptr,P);

end;

The program minreg operates on an arithmetic expression stored as a btree named $E$ with a two-field record at each node and a known maximum height. The question marks on the btree parameters $r$ and maxht indicate that those are implicit parameters -- that is, they will must, however, be exactly "dany btree which is passed as input. The record field names by first declaring a bnode variable, expt". Minreg produces a queue named $P$ from the tree $E$ initialized to the root of $E$ ), then evaluating to point at nodes in $E$ (exptr is automatically function markweights, and finally producing the register requirements of the subtrees with Note that $P$ is automatically initialized to procedure is set up.

Using $M_{k}$ to denote the result of executing markweights on the tree with root k (e.g.,
$M_{\text {right }}=$ markweights(exp.rightson)), we can write the definition of procedure markweights:

markweights(exp: bnode(?E: btree(?r: record(wt,data: integer), ?maxht: integer)))

returns thiswt: integer

post exp.nodeval.wt $=M_{\text {exp }} \wedge\left(\right.$ isleaf $\left.(\exp ) \supset M_{\text {exp }}=0\right)$

$\wedge$ (-isleaf(exp) $\left.\supset M_{n}=\min \left(\max \left(M_{\text {left }}+1, M_{\text {right }}\right), \max \left(M_{\text {left }}, M_{\text {right }}+1\right)\right)\right)=$
begin local leffwt, rightwt: integer;

if isle af(exp) then thiswt $\leftarrow 0$

etse begin

leftwt $\leftarrow$ markweights(exp.leftson);

rightwt $\leftarrow$ markweights (exp.rightson);

this $w t \leftarrow \min (\max ($ left $w t+1$, rightwt), $\max ($ left $w t$, right $w t+1))$;

end;

exp.nodeval.wt $\leftarrow$ thiswt;

end;

2 In some cast

Both styles are acceptable in Alphard, and no deep significance shal notation for clarity. 


\section{Page 6}

Markweights walks over exp (a bnode which indicates a subtree), setting the $w t$ field at each node to the value described by the algorithm above. The post condition, located after the procedure header, specifies the result of the function. It is the formal description of what must be verified about procedure markweights and consequently a theorem about the use of that procedure. The body of markweight uses bnode functions named isleaf, leftson, rightson, and nodeval. It also refers to the $w t$ field of the record stored as the value at each node. These operations are discussed in detail in the next section.

minregwalk (exp: bnode(?E:btree(?r:record(wt,data:integer),?maxht:integer)), order:queue) post (isleaf(exp) $\supset$ order $=$ order' $\sim$ exp.nodeval.data)

$\wedge\left(W_{\text {left }} \geq W_{\text {right }} \supset\right.$ order $=$ order' $\sim Q_{L} \sim Q_{R} \sim$ exp.nodeval.data $)$

$\wedge\left(W_{\text {left }}<W_{\text {right }}>\right.$ order $=$ order $\sim Q_{R} \sim Q_{L} \sim$ invertop $\sim$ exp.nodeval.data $)$

where $Q_{L}, Q_{R}$ are values which satisfy the post conditions of minregwalk $\left(W_{l e f t},<\right)$, minregwalk $\left(W_{\text {right }},<>\right)$ respectively $=$

begin

if risleaf(exp) then

if exp.leftson.nodeval.wt $\geq$ exp.rightson.nodeval.wt

then begin minregwalk (exp.leftson,order); minregwalk(exp.rightson,order) end

else begin minregwalk(exp.rightson,order);

minregwalk(exp.leftson,order); enq(order, invertop) end;

enq(order,exp.nodeval.data);

end;

Minregwalk concatenates a postfix representation of its first argument (a parse tree) to its second argument (a queue). It lests the weights previously stored at the nodes in order to determine the evaluation order of the subtrees. The program uses the same functions on bnodes as markweights; it also uses a queue, but only performs the enq (enqueue) operation. ${ }^{3}$ The formal definition and verification of queues is given elsewhere [Wulf76]; the usage in minregwalk should be clear.

Given suitable specifications of the functions on bnodes and queves, these two procedures can be shown to satisfy their post conditions. ${ }^{4}$ The post conditions are, in turn, direct expressions of the algorithms given in English. It is straightforward, but neither necessary nor appropriate, to demonstrate that the post conditions express the minimalregister property. The algorithms themselves were acceptable on the strength of the analysis that accompanied them, and nothing would be gained by repeating that analysis for the formulation in the program.

3 The enq function appends its second argument to the queue named by the first argument (i.e., enq $(\mathrm{Q}, \mathrm{e})=\mathrm{Q}$ append e). The queue was created (initially empty) in the toplevel procedure minreg for the purpose of collecting the output.

4 The detailed proofs are standard and would contribute little to this exposition of

Alphard. 
Alphard: Design and Verification of a Tree Handler

Page 7

In the next section we define, implement, and verify btrees and their associated bnodes, showing how the information needed to understand their behavior is kept separate from the

\title{
Definition and Verification of a Form
}

\begin{abstract}
Alphard's data abstraction mechanism is the form a syntactic device for encapsulating a set of data declarations, function definitions, and other information about implementation details while revealing to the user only selected information about the behavior of the in the specification. The programs that the implementation supports the behavior described same way that other languages use the previous section used "btree" and "bnode" in the that we could therefore perform the names: we said that exp was a bnode and assumed that defines btrees and bnodes. The operations on it. In this section we develop the form by the procedures above, but ane definition includes not only the functions actually used abstraction. For example, the form defino enough others to round out the form as a useful tree that minreg manipulates.
\end{abstract}

A form contains three major components. These are the specifications, which provide information to the user about the abstract behavior of the objects being defined, the representation, which defines the concrete data structures used to maintain the objects and which states certain of their properties, and the implementation, which contains the bodies of the operators. Thus the skeleton of the btree form is:

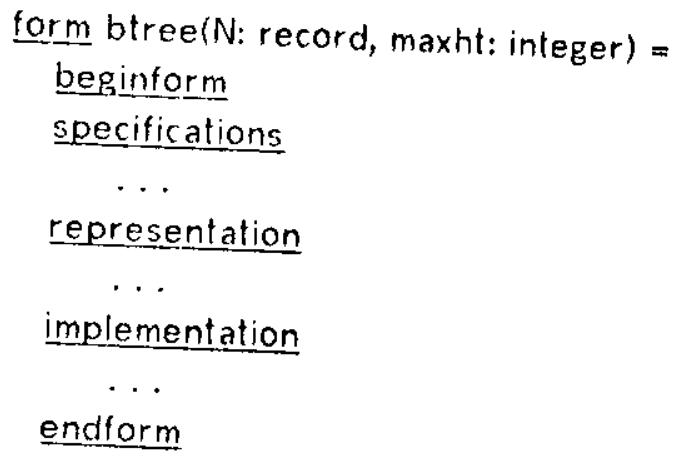

where ellipses are used to denole text which will be filled in later. This form actually record to be stored at each node, $N$, are the maximum height of the btree, maxht, and the that bnodes have also been treated, as "types". Oneters to the instantiation of the form. Note the definition of bnode, which is a formes". One of the components of the btree form is components in turn; the fragments discussed here own right. We will examine each of the in Appendix $A$. 
Page 8

Definition and Verification of a Form

\section{Specifications of btree}

The btree specifications explain what a btree is and how it can be used. They give the restrictions on the instantiation parameters (requires), say that a btree is a special kind of graph $^{5}$ (let, invariant, initially), list the operations that can be performed on it (functions), and give the specifications for bnodes which refer to a given btree (form).

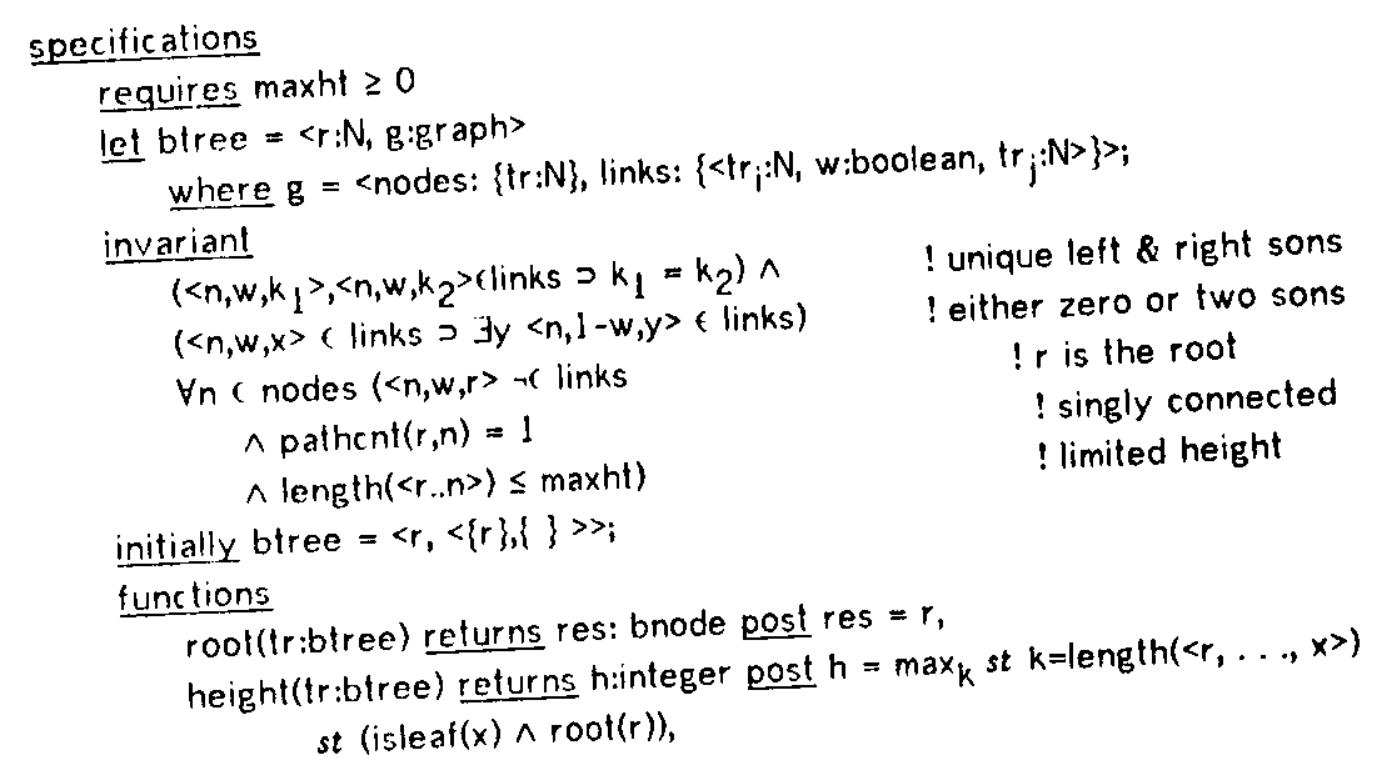

The requires simply says that only nonnegative values of maxht (the maximum height of the tree) make sense. The let declares that a btree may be regarded as a distinguished root and a graph, and that graph concepts will be used to explain them. Since a graph consists of a pair of sets, the let goes on to describe these sets in terms of booleans and the record type passed as an instantiation parameter. The invariant states certain relations on the graph which must always hold of a btree; the comments (! . . ) give the intuitive intertite is empty except for each phrase. Initially states that when a btree is originally instantian name, its input parameters, its the root. For each function, the specifications give the fost conditions needed for verifying the function and result (if any), and the abstract pre and invariant will always be implicitly anded with these and post conditions. The functions root and height are explicit clauses to give the actual pre and applicable to any btree (i.e., any one for which the invariant holds), so the constant true as an explicit pre condition is omitted.

Finally, the btree specifications give the abstract description of the sub-form bnode. The latter form's organization is similar to btree's, except that the specifications of bnode have been printed with those of btree in order to localize the information that will be presented to a user.

5 A suitable definition of graphs is given in Appendix B. 
Alphard: Design and Verification of a Tree Handler

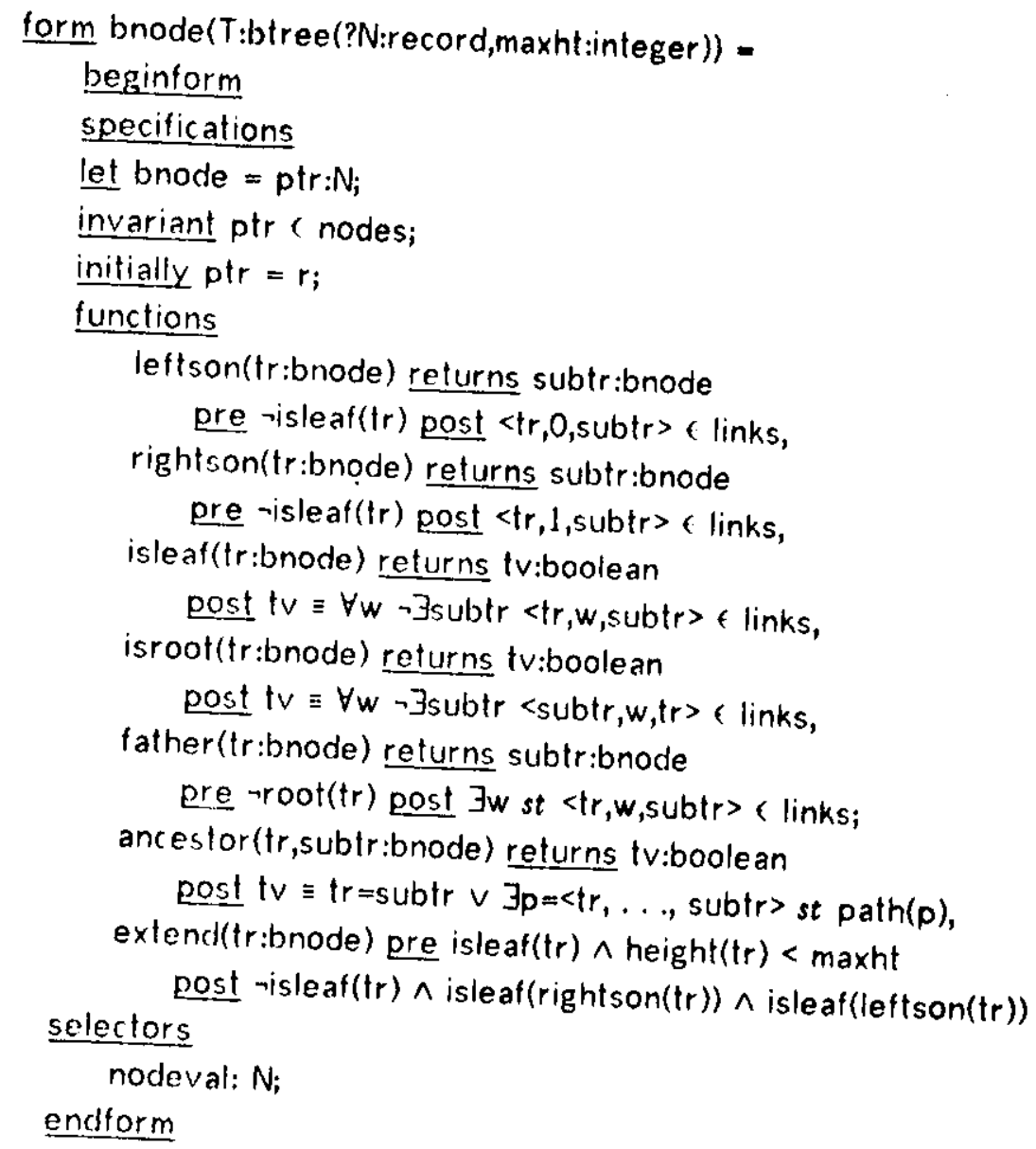

The post conditions of leftson and rightson indicate that a weight of 0 on an arc denotes a left son, while a weight of 1 denotes a right son. The only thing new here is the selectors, which and to fetch values. Note that a bnode is always associated with a particular bired both to set

\section{Representation of btree}

The representation part shows how btrees are actually stored in terms of other data structures (unique, invariant) and explains the correspondence between this concrete representation and the abstract description given in the specifications (rep).

representation

unique $T$ : vector(rec: record(node: $N$, inuse:boolean), $1,2^{\text {maxht }}+1-1$ )

init begin for $x:$ invec $(T)$ do $x$ inuse + false; $T[1]+\operatorname{rec}($ null,true) end;

$\underline{r e p}(T)=\langle T[1]$.node, $<\{T[i]$.node $\mid T[i]$.inuse $\},\{<T[i]$.node, $w, T[2 i+w]$.node $>$

$\mid T[i]$.inuse $\wedge T[2 i+w]$.inuse $\wedge w \in\{0,1\}\}>>$;

invariant $T[1]$.inuse $\wedge(T[i]$.inuse $\supset i=1 \vee T[i$ div 2$]$.inuse $\wedge T[i+1-2(i \bmod 2)]$.inuse); 
The unique declaration states that each btree will consist of a vector of records (node value and "inuse" bit) indexed from 1 to $2^{\text {maxht+1 }}-1$. Alphard's scope rules prevent the vector and the record field names from being used outside the form. The init clause of the declaration gives the initialization code to be executed when that vector is allocated. ${ }^{6}$ it sets all inuse bits to false, then sets the record at the root to (null,true). The unique declaration states that each instance of a btree will get its own vector.

The terms rep(T) and invariant explain how the vector is interpreted as a representation of an abstract tree. The representation function rep $(T)$ exhibits an ordered pair consisting of the node field of $T[1]$, which represents the root, and a pair of sets which represent the graph. The invariant gives a restriction on the distribution of inuse bits which is sufficient to enforce the abstract invariant.

In the representation chosen for this version of btree, all nodes are stored in a vector and the $j^{\text {th }}$ node's sons are found at positions $2 j$ and $2 j+1$. The inuse bit distinguishes whether potential tree positions are actually included in the tree; a separate bit was set aside for this purpose because the node can be an arbitrary record and, as a result, there is no way to encode "nonexistence" in the node value itself. Note that this is the first time a specific implementation strategy has been mentioned: up to this point a linked-list strategy should have seemed equally plausible.

\section{Verification Considerations}

We turn now to the question of how we decide whether a form will actually behave as promised by its abstract specifications -- that is, what properties of a form must be verified if we wish to use its instantiations with confidence. The methodology depends on explicitly separating the description of how an object behaves from the code that manipulates the representation in order to achieve that behavior. It is derived from Hoare's technique for showing correctness of data representations[Hoare72].

The abstract object and its behavior are described in terms of some mathematical entities natural to the problem domain. Graphs are used here to describe btrees; sequences are used in [Wulf 76] to describe queves and stacks, and so on. In btree we appeal to graphs:

- in the invariant, which explains that a btree is a graph that meets certain restrictions,

- in the initially clause, where a particular graph and its root are displayed, and

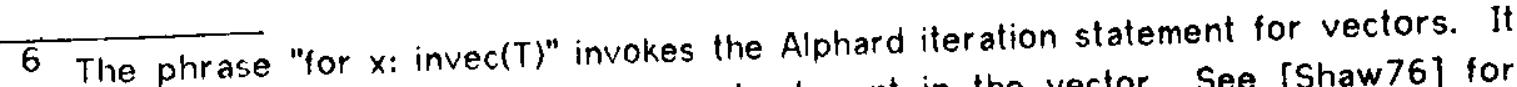
causes the loop to be executed once for each element in the vector. See [Shaw76] for further discussion of iteration. 
Alphard: Design and Verification of a Tree Handler - in the pre and post conditions for each function, which describe the effect the
function has on a graph which satisfies the invariant.

The form contains a parallel set of descriptions of the concrete object and how it behaves. Since btrees are implemented in terms of a vector of records, the concrete of a function muve restrictions and effects on that vector. In many cases this makes the effect

Now, although it is useful to distinguish between the behavior we want and the data structures we operate on, we also need to show a relationship that holds between the two. This is achieved with the representation function rep $(T)$, which gives a mapping from a vector of records to a graph and its root. The purpose of a form verification is to ensure that the two invariants and the rep $(T)$ relation between them are preserved.

In order to verify a form we must therefore prove four things. Two relate to the representation itself and two must be shown for each function. Informally, the four required
steps are ${ }^{7}$ :

For the form

1. Representation validity

$$
I_{c}(T) \supset I_{a}(\operatorname{rep}(T))
$$

2. Initialization

$$
\text { requires }\{\text { init clause }\} \text { initially }(\operatorname{rep}(T)) \wedge I_{c}(T)
$$

For each function

3. Concrete operation

$\underline{i n}(T) \wedge I_{c}(T)\{$ function body $\}$ out $(T) \wedge I_{c}(T)$

4. Relation between abstract and concrete

$$
\begin{aligned}
& \text { 4a. } I_{c}(T) \wedge \operatorname{pre}(\operatorname{rep}(T)) \supset \operatorname{in}(T) \\
& \text { 4b. } I_{c}(T) \wedge \operatorname{pre}\left(\operatorname{rep}\left(T^{\prime}\right)\right) \wedge \operatorname{out}(T) \supset \operatorname{post}(\operatorname{rep}(T))
\end{aligned}
$$

Step 1 shows that any legal state of the concrete representation has a corresponding abstract object (the converse is deducible from the other steps). Step 2 shows that the initial state created by the representation section is legal. Step 3 is the standard verification formula for the concrete operation as a simple program; note that it enforces the preservation of $\mathrm{I}_{\mathrm{c}}$. Step

7 We will use $\mathrm{I}_{\mathrm{a}}(\mathrm{rep}(\mathrm{T}))$ to denote the abstract invariant of an object whose concrete formulas. In step 4b, "pre(rep (T')" specification clauses and assertions to refer to those A complete development of the form vefers to the value of T before execution of the function. 
4 guarantees (a) that the concrete operation is applicable whenever the abstract pre condition holds and (b) that if the operation is performed, the result corresponds properly to the abstract specifications.

For btree, several of these steps will be simplified by appealing to the following standard construction, which determines the correspondence between an index in the vector representation and a path from the root to a node in the abstract graph.

Let $T[j]$ be the vector element which represents some node in a btree.

Let $w_{0} w_{1} w_{2} \ldots w_{k}$ be the binary representation of $j, w_{0}=1$.

Define $p_{i}$ as $p_{i}=S U M_{b}=0 \ldots i\left(w_{b} 2^{i-b}\right)$ for $i=0 \ldots k$ (note that $p_{i}=2 p_{i-1}+w_{i}$ and $p_{k}=j$ ).

Then the (abstract) path from the root to a node is the path whose elements are $<T\left[p_{i-1}\right]$.node, $w_{i}, T\left[p_{i}\right]$.node $>$ for $i=1 \ldots k$

In addition, if the node is in the tree, T[j].inuse = true and, because of the term $T[i]$.inuse $\supset i=1 \vee T[i$ div2 $]$.inuse

of the invariant, all elements in the path are also in the tree.

\section{Verification of form properties of btree}

At this point we have enough information about btrees to perform verification steps 1 and 2 , which show the overall validity of the form. We can now proceed with an informal proof of these steps.

1. Representation Validity

Show: $T[1]$.inuse $\wedge(T[i]$.inuse $\supset i=1 \vee T[i$ div 2].inuse $\wedge T[i+1-2(i \bmod 2)]$.inuse $) \supset$ $\left(\left\langle n, w, k_{1}\right\rangle,\left\langle n, w, k_{2}\right\rangle\left(\right.\right.$ links $\left.\supset k_{1}=k_{2}\right) \wedge(\langle n, w, x\rangle \in$ links $\supset \exists y\langle n, 1-w, y\rangle \in$ links $)$ $\forall n<$ nodes $(<n, w, r>\sim$ links $\wedge$ pathent $(r, n)=1 \wedge$ length $(<r . . n>) \leq$ maxht $)$ where nodes $=\{T[i]$.node $\mid T[i]$.inuse $\}$

links $=\{\langle T[i]$. node, $w, T[2 i+w]$. node $>| T[i]$.inuse $\wedge T[2 i+w]$.inuse $\wedge w \in\{0,1\}\}$

Proof: Take the clauses of the conclusion one by one:

(a) $k_{1}=k_{2}$ bec ause the rep function uniquely determines the triples in links on the basis of $n$ and $w$.

(b) $\exists y\langle n, 1-w, y\rangle$ because both sons or neither son of a node have the inuse bit set.

(c) $\langle n, w, r\rangle \rightarrow$ links because $r=T[1]$.node and $1 \neq 2 i+w$ for any integer $i \geq 1$.

(d) pathent $(r, n)=1$ because the standard construction is unique.

(e) length $(\langle r . . n\rangle) \leq$ maxht because each vector index must be in the range $\left[1.2^{\text {maxht+1 }}-1\right]$ and the standard construction gives a path whose length is the number of significant bits in the vector index. 


\title{
Alphard: Design and Verification of a Tree Handler
}

2. Initialization

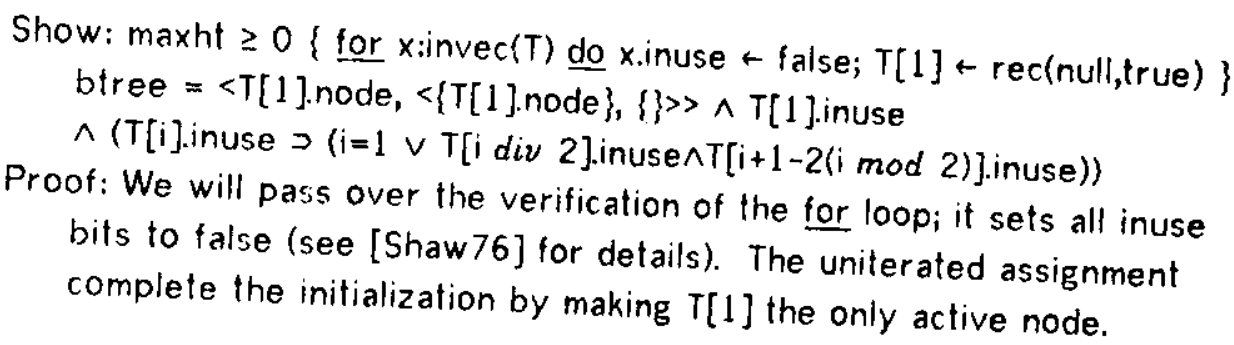

These steps demonstrate that any vector $T$ which satisfies $I_{c}$ represents a legal limited-height btree and that the initial value of a newly-instantiated btree is initialized properly. We will adequacy of that representation is established here.

\section{Implementation of btree}

The implementation part gives the bodies of the two functions and the bnode form promised by the specifications. For each function, we provide both the program to compute the function and the concrete in and out conditions. Although neither function is used in the minreg program, they are included in the btree form in order to make it a more generally is illustrated below for functions we have actually used.

\section{implementation}

body root out res $=1=$

! The bnode return parameter is initialized to the root.

body height out $h=\log \left(\max _{i}\right.$ st $T[i]$.inuse $)=$

first j: downto $\left(2^{\text {maxht }+1}-1,1\right)$ suchthat $T[j]$.inuse

$$
\text { then } h \leftarrow \text { floor }\left(\log _{2} j\right) \text {; }
$$

\section{Implementation of bnode}

The bnode form is organized like the btree form, and its verification proceeds in a

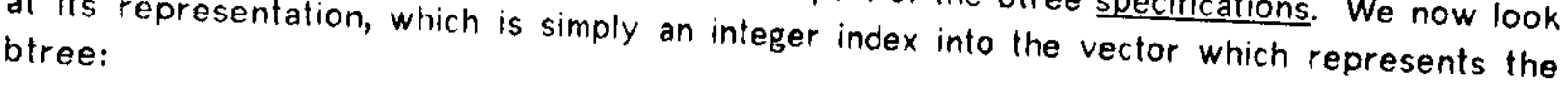

\author{
representation \\ unique ptr: integer init ptr $\leftarrow 1$; \\ rep $(p t r)=T[p t r] \cdot$ node; \\ invariant $1 \leq p t r \leq 2^{\text {maxht }+1}-1 \wedge T[p t r]$.inuse;
}


To verify the form properties, we must prove two things:

1. Representation validity

Show: $1 \leq$ ptr $\leq 2^{\text {maxht }+1}-1 \wedge T[$ ptr].inuse $\supset T[p t r]$.node $\in\{T[i]$.node $\mid T[i]$.inuse $\}$

Proof: Clear.

2. Initialization

Show: true $\{$ ptr -1$\} T[p t r] \cdot$ node $=T[1]$ node $\wedge 1 \leq p t r \leq 2^{\text {maxht+1}}-1$

$\wedge T[p t r]$ inuse

Proof: Applying the rep function and the assignment axiom, this becomes

$T[1]$.node $=T[1]$.node $\wedge 1 \leq 1 \leq 2^{\text {maxht }+1}-1 \wedge T[1]$.inuse

This reduces to $T[1]$.inuse, which is assured by the concrete invariant of btree.

Thus we have shown that the representation supports the abstraction. We will next discuss and verify some of the functions used by the programs of the previous section. Other functions are given in the form definition in Appendix A. Note that the invariants of btree (as well as those of bnode) must be preserved. This step is omitted from the proofs given here because no part of the btree representation is altered.

One of the simplest functions finds the left son of a given node. Its abstract specifications and body are:

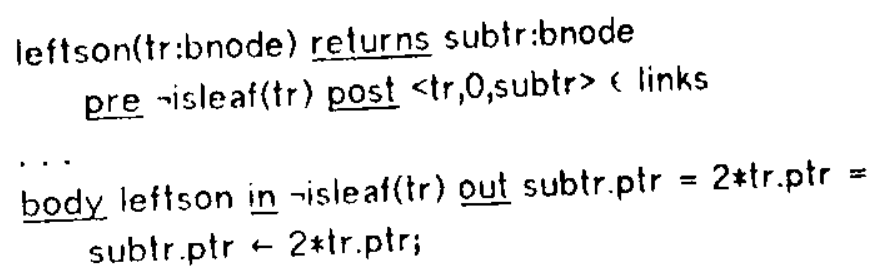

The program itself is clear: double a node's index to find its left son. The in condition asserts that the leftson function may not be applied to a leaf 8 The out condition repeats the doubling property. Recall that the concrete invariant must be shown to hold along with the in and out conditions, so we may be sure leftson is applied only to legal bnodes and does not destroy them. These properties are verified formally by proving the following (again $I_{c}$ denotes the concrete invariant):

8 This design decision forces the user to extend the tree explicitly before using new nodes, but it offers a degree of protection against errors that automatic tree growth would not. We could, of course, extend the tree automatically when leftson or rightson is applied to a leaf, but that is a different decision and leads to a different program. 
3. Concrete operation

Show: $\exists j\left(j\right.$ div $2=\operatorname{tr} . p t r \wedge \operatorname{tr} . T[j]$.inuse $\left.\wedge 1 \leq j \leq 2^{\text {maxht }+1}-1\right) \wedge I_{c}$

Proof: Choosing $j=2 *$ tr.ptr and applying $=2 * \operatorname{tr} . p t r \wedge \mathrm{I}_{c}$

tr.ptr $=$ tr.ptr $\wedge$ tr.

$\supset 2 * t r . p t r=2 * \operatorname{tr} . p t r \wedge I_{c}$

The concrete invariant for $t r$ is maintained since tr is not modified; it is established for subtr because of the range check on $j$.

4a. in condition holds

Show: $1 \leq t r . p t r \leq 2^{\text {maxht }+1}-1 \wedge$ tr.T[tr.ptr].inuse $\wedge \exists w, s<t r, w, s>$ elinks

$\supset \exists j$ ( $j$ div $2=\operatorname{tr}$.ptr $\wedge$ tr. T[j].inuse $\wedge 1 \leq j \leq 2^{\text {maxht }+1}-1$ )

Proof: If $\exists w, s$ st $\langle t r, w, s\rangle$ (links, then s must correspond to the vector element inclexed by $2 * t r . p t r+w$, and it must be an active node. This is sufficient to
establish the conclusion.

4b. post condition holds

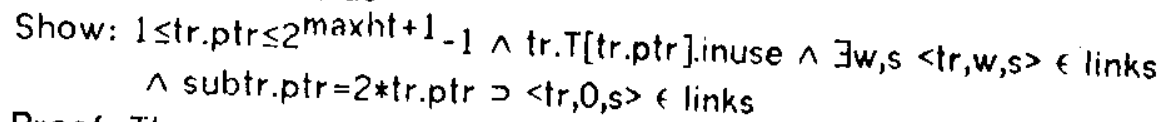

Proof: The concrete invariant of btree says that the s must be $2 * t r . p t r+w$ and that both $\langle t r, 0, s\rangle$ and $\langle t r, 1, s\rangle$ exist, which is

precisely the condition needed.

Note that the proof refers to both the ptr field of the input parameter $t r$ and the tree $T$ for which $\mathrm{tr}$ was created. Qualified names may be used for this selection, so we write tr.ptr and
tr.T, respectively. These phrases can be further element of vector tr.T by writing tr.T[i] (since $T$ is qualified, so we can select a particular that vector element by writing tr.T[i].inuse. The definition and verification of essentially the same.

We often needed to determine whether the tree we had in hand was a leaf. The specifications and function body for isleaf are

$$
\begin{aligned}
& \text { isleaf(tr:bnode) returns tv:boolean } \\
& \text { post } t v \equiv \forall w-\exists \text { subtr }\langle t r, w, s u b t r>\epsilon \text { links } \\
& \begin{array}{l}
\text { out } t \vee \equiv\left(-\exists j\left(j d i v 2=\operatorname{tr} . p t r \wedge t r . T[j] . \text { inuse } \wedge 1 \leq j \leq 2^{\text {maxht }+1}-1\right)\right)= \\
t \vee \leftarrow \operatorname{tr} . p t r>2^{\text {maxht }}-1 \vee(-t r . T[2 * t r . p t r] . i n u s e \wedge \rightarrow t r . T[2 * t r . p t r+1] \text {.inuse }) ;
\end{array}
\end{aligned}
$$

The out condition specifies that isleaf returns "true" if there is no vector index in range for which $T[j]$ is both in use and a left or right son of the input. Since the in condition is omitted, it is assumed to be identically true, so isleaf must be applicable to any btree. To verify isleaf,
we must show the following: 


\section{Page 16}

3. Concrete operation

Show: $I_{c}\left\{t v \leftarrow t r . p t r>2^{\text {maxht }}-1 \vee(-t r . T[2 * t r . p t r]\right.$.inuse $\wedge-t r . T[2 * t r . p t r+1]$.inuse $\left.)\right\}$ $\left(t \vee \equiv-\exists j\left(j\right.\right.$ div $2=\operatorname{tr}$.ptr $\wedge$ tr. T[j].inuse $\left.\wedge 1 \leq j \leq 2^{\operatorname{maxht}+1}-1\right]$.inuse $\left.)\right) \wedge I_{c}$ Proof: Rewriting to eliminate $j$ and applying the assignment axiom, this becomes

$$
\begin{aligned}
I_{C} & \supset\left[\left(1 r . p t r>2^{\text {maxht }}-1 \vee(-t r . T[2 * t r . p t r] . \text { inuse } \wedge-t r . T[2 * t r . p t r+1] . \text { inuse })\right.\right. \\
& \equiv-\left(( t r . T [ 2 * \operatorname { t r } . p t r ] \text { .inuse } \vee \operatorname { t r } . T [ 2 * t r . p t r + 1 ] \text { .inuse } ) \wedge \left(1 \leq 2 * t r . p t r \leq 2^{\text {maxht }+1}-1\right.\right. \\
& \left.\left.\left.\left.\vee 1 \leq 2 * t r . p t r+1 \leq 2^{\text {maxht }+1}-1\right)\right) \wedge I_{c}\right)\right]
\end{aligned}
$$

which in turn reduces to

$\mathrm{I}_{\mathrm{c}} \supset\left[\left(t r . p t r>2^{\text {maxht }}-1 \vee(-t r . T[2 * t r . p t r]\right.\right.$.inuse $\wedge-t r . T[2 * t r . p t r+1]$.inuse $)$

$\equiv-\left((t r . T[2 * t r . p t r]\right.$.inuse $\vee \operatorname{tr} . T[2 * t r . p t r+1]$.inuse $) \wedge\left(1 \leq t r . p t r \leq 2^{\text {maxht }}\right.$

$\vee 1 \leq$ ir.ptr $\left.\left.+1 \leq 2^{\text {maxht })} \wedge I_{c}\right)\right]$

which is clear.

4a. in condition holds

Show: $I_{c} \supset$ true

Proof: Clear.

4b. post condition holds

Show: $l_{c} \wedge t v \equiv-j j\left(j\right.$ div $2=\operatorname{tr}$.ptr $\wedge \operatorname{tr} . T[j]$.inuse $\left.\wedge 1 \leq j \leq 2^{m a x h t+1}-1\right]$.inuse $)$

$$
\supset \forall w-\exists \text { subtr }(<t r, w, \text { subtr }>\text { (links) }
$$

Proof: The out says there is no $w$ for which $T[t * t r . p t r+w]$.inuse, either because

$2 * t r$.ptr would exceed the index range of the array or because the inuse

bit is set to false. By the definition of links, there is no triple

$<t r . T[t r . p t r], w, t r . T[2 * t r . p t r+w]>$ which could correspondto $\langle t r, w, s u b t r>$.

Finally, bnode provides a selector, nodeval, for performing fetches and stores to the value field of a tree node. The implementation of nodeval is given by

map nodeval $=T[$ ptr $]$.node;

Changing this particular field has no effect on any invariant, so nothing must be proved.

\section{Conclusion}

This paper has used a concrete example to explain the Alphard philosophy on the development and verification of programs. The example was nontrivial; it implemented the abstraction with a nonstandard representation, and it involved a subtype. Several aspects of the development deserve special notice. 
Alphard: Design and Verification of a Tree Handler

Page 17

First, note that we did not verify the "main program". The program was simply a restatement of an algorithm that had undergone considerable analysis in another formulation. We therefore been unreasonable to redo that analysis in the course of verifying the program. restatement of the algorithm. take such steps to avoid reproving verification is ever to impact real programs, we must encapsulates a collection of related all programs from first principles. Since the form achieved, if is a reasonable body of evidenced by the nearly complete independenation about which to prove theorems. This is the btree form.

Next, the form presented in Appendix A contains functions not actually used by the program of the example. We believe that in the future libraries of forms will develop, and that verification considerations than present libraries because the forms are verified and because abstraction. Further, the explicit distinction thought about what constitutes a good concrete implementation should simplify modification of the abstract specification and the on which users depend are made clear and also of the code both because the assumptions have to be repeated.

Finally, some of our colleagues have expressed concern over the length of Alphard programs. Certainly the verification information adds text, but we believe that this information must be supplied somewhere. Nakata gave an Algol program for converting a parse tree to code [Nakata67]. That program performs a slightly different operation from minreg, so an functions that were never used, the is within 107. of the number of lexemes number of lexemes in the Alphard procedures and forms our feeling that the program text itsell is Nakata's program. This crude comparison supports

\section{Acknowledgements}

The abstractions, programs, and verifications presented here have benefited from comments by a number of my colleagues in the Alphard project, particularly Bill Wulf and
Ralph London. 


\title{
Appendix A \\ Complete Definition of Btree and Bnode
}

\author{
form btree(N:record, maxht:integer $)=$ \\ beginform \\ specifications
}

requires maxht $\geq 0$

let btree $=\langle r: N, g: g r a p h\rangle$

where $G=\left\langle\right.$ nodes: $\{t r: N\}$, links: $\left\{\left\langle t r_{i}: N\right.\right.$, w:boolean, $\left.\left.\operatorname{tr}_{j}: N>\right\}\right\rangle ;$

invariant

$$
\begin{array}{lc}
\left(<n, w, k_{1}>,<n, w, k_{2}>\left(\text { links } \supset k_{1}=k_{2}\right) \wedge\right. & \text { ! unique left \& right sons } \\
(<n, w, x>(\text { links } \supset \exists y<n, 1-w, y>\epsilon \text { links }) & \text { ! either zero or two sons } \\
\forall n<\text { nodes }(<n, w, r>\rightarrow \text { links } & \text { ! } r \text { is the root } \\
\wedge \text { pathent }(r, n)=1 & \text { ! singly connected } \\
\wedge \text { length }(<r . . n>) \leq \text { maxht }) & \text { ! limited height }
\end{array}
$$

initially btree $=\langle r,\langle\{r\},\{\}\rangle\rangle$;

functions

root(tr:btree) returns res: bnode post res $=r$,

height(tr:btree) returns $h$ :integer post $h=\max _{k}$ st $k=$ length $(\langle r, \ldots, x\rangle)$

$$
\text { st }(\text { isleaf }(x) \wedge \operatorname{root}(r)) \text {, }
$$

form bnode (T:btree(?N:record, maxht:integer)) $=$

beginform

specifications

let bnode = ptr:N;

invariant ptr $\mathrm{f}$ nodes;

initially ptr $=r$;

functions

leftson(tr:bnode) returns subtr:bnode

pre -isle af(tr) post <tr,0,subtr> c links,

rightson(tr:bnode) returns subtr:bnode

pre -isleat(tr) post $\langle t r, 1$,subtr $<$ links,

isleat(tr:bnode) returns tv:boolean

post $t v \equiv \forall w-\exists$ subtr $<t r, w$,subtr $>\in$ links,

isroot(tr:bnode) returns tv:boolean

post tv $\equiv \forall w-3$ subtr <subtr, $w, t r>$ \& links,

father(tr:bnode) returns subtr:bnode

pre $\rightarrow r \cot (t r)$ post $\exists w$ st $\langle t r, w, s u b t r>$ ( links;

ancestor(tr,subtr:bnode) returns tv:boolean

post tv $\equiv t r=s u b t r \vee \exists p=<t r, \ldots$, subtr $>$ st path $(p)$,

extend(tr:bnode) pre isleaf(tr) $\wedge$ height $(t r)<$ maxht

post -isleaf $(t r) \wedge$ isleat(rightson(tr) $) \wedge$ isleaf(leftson $(t r))$ 
selectors

nodeval: $\mathrm{N}$;

endform

\section{representation}

unique $T$ : vector(rec: record(node: $N$, inuse:boolean), $1,2^{\text {maxht }+1}-1$ )

init begin for $x$ invec $(T)$ do $x$ inuse $\leftarrow$ false; $T[1] \leftarrow$ rec(null,true) end;

$\underline{\operatorname{rep}}(T)=\langle T[1]$. node, $<\{T[i]$. node $\mid T[i]$.inuse $\},\{<T[i]$.node, $w, T[2 i+w]$. node $>$

$\{T[i]$.inuse $\wedge T[2 i+w]$.inuse $\wedge w \in\{0,1\}\}>>_{i}$

invariant $T[1]$.inuse $\wedge(T[i]$.inuse $\supset i=1 \vee T[i \operatorname{div} 2]$.inuse $\wedge T[i+1-2(i \bmod 2)]$.inuse);

implementation

body root out res $=1=$

! The bnode return parameter is inilialized to the root.

body height out $h=\log (\max ;$ st $T[i]$.inuse $)=$

first j: downto $\left(2^{\text {maxht }+1}-1,1\right)$ suchthat $T[j]$.inuse

then $h \leftarrow$ floor $\left(\log _{2} j\right)$;

formbody bnode $=$

beginform

representation

unique ptr: integer init ptr $\leftarrow 1$;

$\underline{r e p}(p t r)=T[p t r]$ node;

invariant $1 \leq p t r \leq 2^{\text {maxht }+1}-1 \wedge T[p t r]$.inuse;

implementation

body leftson in -isleaf(tr) out subtr.ptr $=2 *$ tr.ptr $=$

subtr.ptr $\leftarrow 2 * t r$.ptr;

body rightson in -isleaf(tr) out subtr.ptr $=2 * t r . p t r+1=$ subtr.ptr $\leftarrow 2 *$ tr.ptr +1 ;

body isteaf

out $t \vee \equiv\left(-\exists j\left(j\right.\right.$ div $2=\operatorname{tr} . p t r \wedge \operatorname{tr} . T[j]$.inuse $\left.\left.\wedge 1 \leq j \leq 2^{\operatorname{maxht+1}}-1\right)\right)=$ t $\vee \leftarrow$ tr.ptr $>2^{\text {maxht }}-1 \vee(-t r . T[2 * t r . p t r]$.inuse $\wedge-t r . T[2 * t r . p t r+1]$.inuse);

body isroot out tv $\equiv($ tr.ptr $=1)=$

$t v \leftarrow$ tr.ptr $=1$;

body father in tr.ptr $>1$ out subtr.ptr $=\operatorname{tr}$.ptr div $2=$ subtr.ptr $\leftarrow$ tr.ptr div 2 ; 
Page 20

Appendix A

body ancestor in $\operatorname{tr} . T[t r . p t r]$.inuse $\wedge$ tr.T[subtr.ptr].inuse out $i v \in\left(\exists j_{1}, j_{2}, \ldots j_{k}\right.$ st $j_{1}=$ tr.ptr $\wedge j_{k}=$ subtr.ptr

$\wedge \operatorname{tr} . T\left[j_{i}\right]$.inuse $\wedge j_{i-1}=j_{i}$ div 2) $=$

begin local shftd;

shitd $\leftarrow$ floor $\left(\log _{2}\right.$ subtr.ptr $)-$ floor $\left(\log _{2}\right.$ tr.ptr $)$;

tv $\leftarrow$ tr.ptr $=$ subtr.ptr div $2^{\text {shitd; }}$ end;

body extend in isleaf(tr.ptr) $\wedge$ tr.ptr $<2^{\text {maxht }}$

out -isleaf(tr.ptr) $\wedge$ isleat(rightson(tr.ptr)) $\wedge$ isleaf(leftson(tr.ptr)) $=$

begin

$\operatorname{tr} . T[2 * t r . p t r]$.inuse $\leftarrow \operatorname{tr} . T[2 * t r . p t r+1]$.inuse $\leftarrow$ true;

$\operatorname{tr} . T[2 * t r . p t r] . n o d e+\operatorname{tr} . T[2 * t r . p t r+1]$.node $\leftarrow$ null;

end;

map nodeval $=T[p t r]$.node;

endform;

endform; 
Alphard: Design and Verification of a Tree Handler

Page 21

\section{Appendix B
Formal Definition of Graphs with Weighted Arcs} This formal definition is based on the definition of graph given by Knuth [Knuth73, sec. 2.3.4]
with the addition of labels, or weights, on the arcs. 1. Let $N$ be a set called the node domain of a graph and let $W$ be a set called the
arc weights of a graph.

(a) An arc is a triple $\left\langle n_{i}, w_{j}, n_{k}>\right.$ where $n_{i}\left(N, w_{j} \in W, n_{k} \in N\right.$

(b) A graph is a pair $\langle E, A\rangle$ where $E$ is a set of nodes and $A$ is

a set of arcs such that $\left\langle n_{j}, w_{j}, n_{k}>\left\langle A \supset n_{i}, n_{k}<E\right.\right.$

(c) These are the only graphs. 2. The notation $\left\langle n_{1}, n_{2}, \ldots, n_{k}\right\rangle$ is an abbreviation for $\left\{\left\langle n_{1}, w_{1}, n_{2}\right\rangle,\left\langle n_{2}, w_{2}, n_{3}\right\rangle\right.$,
$\left.\ldots,\left\langle n_{k-1}, w_{k-1}, n_{k}\right\rangle\right\}$ for any values of $w_{j}$

3. The following functions and relations are defined for $G=\left\langle E, A>\right.$ and $n_{j} \in E$ :

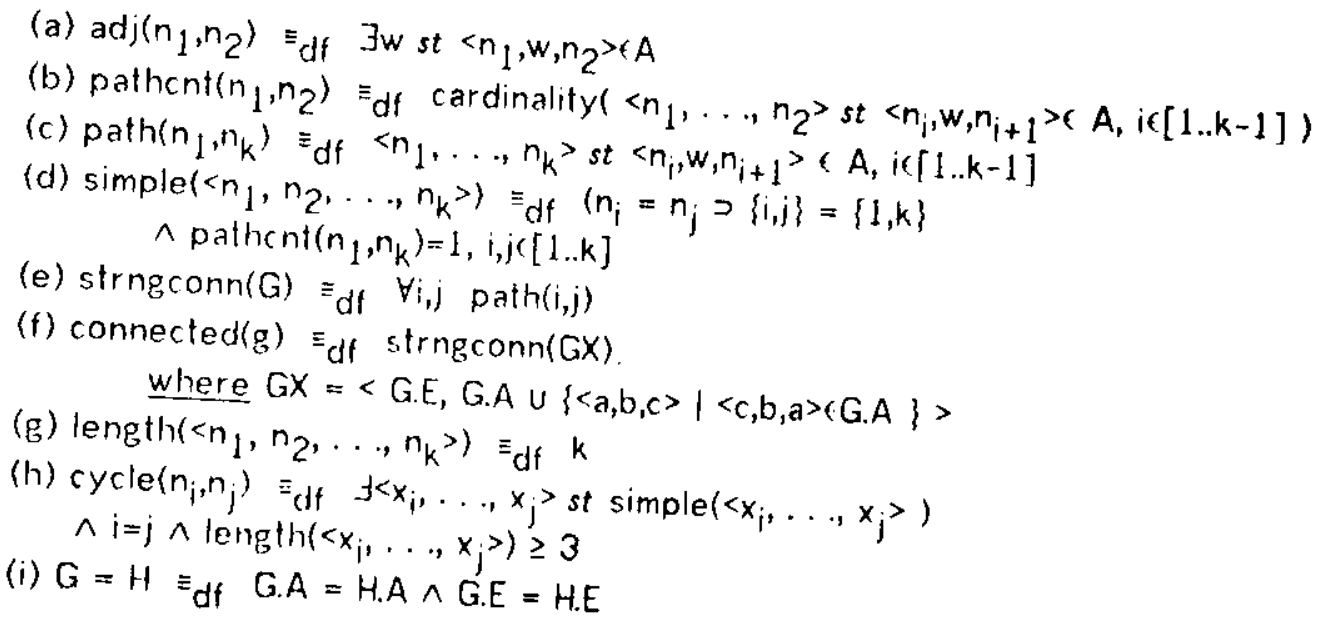




\section{Appendix B}

Page 22

\section{References}

[Dahl72] 0.-J. Dahl and C. A. R. Hoare, "Hierarchical Program Structures", in Structured Programming (Dahl, Dijkstra, and Hoare), Academic Press, 1972 (pp. 175-220).

[Dijkstra72] Edsger W. Dijkstra, "Notes on Structured Programming", in Structured Programming (Dahl, Dijkstra, and Hoare), Academic Press, 1972 (pp. 1-82).

[Hoare72] C. A. R. Hoare, "Proof of Correctness of Data Representations", Acte Informatica, $1,4,1972$ (pp. 271-281).

[Johnsson75] Richard K. Johnsson, "An Approach to Global Register Allocaton", CarnegieMellon University Technical Report, December 1975.

[Knuth73] Donald E. Knuth, Fundamental Algorithms, Second Edition, Addison-Wesley, 1973.

[Liskov74] Barbara Liskov and Stephen Zilles, "Programming with Abstract Data Types", SIGPLAN Notices, 9,4, April 1974 (pp.50-59).

[Nakata67] Ikuo Nakata, "A Note on Compiling Algorithms for Arithmetic Expressions", Communications of the ACM, 10,8, August 1967.

[Parnas 72] David L. Parnas, "On the Criteria to be Used in Decomposing Systems into Modules", Communications of the ACM, 15, 12, December 1972 (pp. 1053-1058).

[Shaw 76] Mary Shaw, William Wulf, and Ralph L. London, "Abstraction and Verification in Alphard: Iteration and Generators", Carnegie-Mellon University Technical Report and USC Information Sciences Institute Research Report, 1976.

[Wirth71] Niklaus Wirth, "Program Development by Stepwise Refinement", Communications of the ACM, 14, 4, April 1971 (pp. 221-227).

[Wulf76] William Wulf, Ralph L. London, and Mary Shaw, "Abstraction and Verification in Alphard: Introduction to Language and Methodology", Carnegie-Mellon University Technical Report and USC Information Sciences Institute Research Report, 1976. 